\title{
Improving the efficiency of fixed radionuclides' removal by chemical decontamination of surfaces in situ*
}

\author{
Yuriy S. Koryakovskiy ${ }^{1}$, Valeriy A. Doilnitsyn ${ }^{1}$, Andrey A. Akatov ${ }^{1}$ \\ 1 Saint-Petersburg State Institute of Technology (Technical University), 26 Moskovsky prospekt, St. Petersburg, 190013, Russian Federation \\ Corresponding author: Yuriy S. Koryakovskiy (yuriy226@mail.ru)
}

Academic editor: Yury Korovin • Received 8 March 2019 • Accepted 18 May 2019 Published 21 June 2019

Citation: Koryakovskiy YS, Doilnitsyn VA, Akatov AA (2019) Improving the efficiency of fixed radionuclides' removal by chemical decontamination of surfaces in situ. Nuclear Energy and Technology 5(2): 155-161. https://doi.org/10.3897/nucet.5.36477

\begin{abstract}
The article presents the results of work aimed at improving of chemical decontamination methods. A brief description of existing chemical decontamination technologies used to remove radioactive contamination (RC) from walls, floors and external surfaces of equipment without dismantling, i.e., in situ, is given. A vector of research aimed at improving the efficiency of fixed RC's removal is also determined. The first aim of this work is to improve the decontaminating properties of removable polymer coatings used in practice. The following domestic products were chosen as study objects: compositions presented under trademarks VA, VL; and also special formulation ZPS-1M. Modifications of these compounds performed in SPSIT in some cases made it possible to significantly increase the decontamination factors (DF). The best results were obtained for VL compositions: it was found that due to certain additives it is possible to increase the DF for metal surfaces by a factor of 5-35 over the base product. Along with film-forming decontaminating compositions, an alternative patented technique has been developed in SPSIT. The main feature of this technique is usage of sorbent-based composite covering material previously saturated by decontaminating solution. New technique allows to achieve far higher decontamination factors (150-500) when fixed RC is removed from metal surfaces. In addition, it can be applied to polymer and other non-metal materials. One of the main advantages of given technique is a drastic (11-16 times) reduction of time required for carrying out decontamination operations. The obtained results may be useful for further research in this area. Thus, research performed allows to come up with general conclusion: there are possibilities to efficiently remove fixed RC from surfaces using rather simple chemical means. That, in its turn, could be a rational alternative to high-priced robotic decontaminating systems.
\end{abstract}

\section{Keywords}

decontamination, decommissioning, fixed radioactive contamination, film-forming compositions, composite decontamination material

\section{Introduction}

By now, many radiation-hazardous facilities (nuclear power units, radiochemical production facilities, research sites, etc.) have had their service life expired and require decommissioning. Decommissioning of a major facility normally suggests a huge amount of decontamination activities.

Decontamination in the process of decommissioning has the purpose to release for unrestricted reuse as many materials as possible to reduce the risk of dangerous exposure for personnel involved in decontamination, as well 
as to avoid the RC spreading (specifically, with radioactive dust) into other rooms and over the site.

During decontamination, both decontamination methods used to process dismantled (fragmented) equipment in special areas (for instance, decontamination of scrap metal in baths) and procedures used for the RC removal from walls, floors and lining of chambers and boxes, as well as from the outer surfaces of equipment without disassembly (in situ) are of importance.

Among latter one can distinguish liquid decontamination methods, e.g., jet treatment of the contaminated surface with water or a chemical reagent solution. These methods have been used earlier on rather a broad scale but there has been a later trend towards reducing the amounts of RW resulting from decontamination. By now, therefore, there are a number of the so-called dry and low-waste technologies as an alternative to liquid decontamination methods leading to the maximum amount of secondary radioactive waste (Vasilenko et al. 2010).

Some of the criteria should be taken into account to select the decontamination method:

- high efficiency of RC removal;

- no RC spreading;

- smallest possible amount of secondary RW;

- cost effectiveness;

- explosion and fire safety, etc. (Koryakovskiy et al. 2010).

The above requirements are valid also for in-situ local decontamination technologies. Besides, methods in this group are expected to ensure, where possible, that the personnel involved in the process of cleaning stay in the hazardous area (characterized by an increased dose rate and/or a high aerosol activity) for as short time as possible. The implementation of this term makes it possible to minimize the personnel exposure.

The following technologies can be identified among the chemical methods fit for in-situ decontamination:

- wiping with rags wetted with a decontamination solution;

- wet vacuum treatment (when the vacuum cleaner is charged with a decontamination solution);

- electrochemical decontamination with use of external electrode;

- foam decontamination;

- decontamination by etching pastes and gels;

- decontamination by removable polymer coatings;

- decontamination by sorbents.

Each of the above methods has drawbacks of its own, e.g. vacuum cleaners, sorbents, commercially available foam compounds and film-forming compositions turn out to be not so efficient in most cases when it comes to removal of fixed radioactive contamination. Fixed RC means contamination that has a strong bond with the oxide film on the metal surface or has penetrated into the metal, or, in the event of polymer materials, $\mathrm{RC}$ that has diffused into the depth of the polymer (e.g. paint coatings).

Wiping with rags soaked with a formulation based on strong mineral acids, application of etching pastes and gels (Ampelogova et al. 1982, RF Patent No. 2259422, RF Patent No. 2017244, Pat. of WIPO WO2013092633), or off-bath electrochemical decontamination (with an external electrode in the form of a brush having holes for the forced supply and extraction of the electrolyte (Ampelogova et al. 1982, Kritskiy 2010)) can be recommended for the removal of fixed RC from metal surfaces. However, these methods have a low operation rate which is a drawback. Thus, wiping, and application of pastes and gels to the surface, and, especially, the removal of these gels require quite a time. For example, the spreading of an even paste layer over the contaminated metal surface requires about 20 to 30 seconds per $\mathrm{m}^{2}$; their removal requires further 45 to $90 \mathrm{~s}$, and, quite often, still more is taken to wash the agent residues off the metal. Still less productive is an electric brush with which the treatment rate is about 8 to $10 \mathrm{~min} / \mathrm{m}^{2}$ (for current density about 250 to $700 \mathrm{~A} / \mathrm{dm}^{2}$ and the electrode area $1 \mathrm{dm}^{2}$ (Ampelogova et al. 1982)). And the increase of the work time in the contaminated area increases the risk of the personnel overexposure.

The above factors lead to the understanding of need for such methods and means for external in-situ decontamination which would make it possible to remove fixed RC in an efficient manner with the shortest possible personnel exposure time.

Of interest from this point of view are removable polymer coatings. For the first time in domestic practice these decontamination means were tested on radioactively hazardous facilities of the Navy (coastal maintenance bases and nuclear submarines); in particular, formulations based on polyvinyl alcohol were used on a broad scale. Since such compositions have a short storage period, they had to be prepared right in site directly prior to the use (Koryakovskiy et al. 2014a).

In the 1980s, polymer compositions were used for decontamination during the Chernobyl NPP post-accident recovery activities; predominantly, NIKIMT-developed compositions were used (Koryakovskiy et al. 2014a).

The ZPS-1M agent based on a polyvinyl emulsion was adopted in the early 2000s for application in the Navy; the formulation has the decontaminating action due to the addition of orthophosphoric acid. The separation of the composition into three components (polyvinylacetate dispersion, pigment finish and concentrated orthophosphoric acid) made it possible to provide a five-year shelf life (TU 2316-219-56271024-2007).

Film-forming decontamination compositions, including VA-501, VA-502, VA-503, VA-504, and VLЛ-501, VL-502, VL-503, and VL-504 can be assigned to separate category. The VA grade formulations are based on aqueous solutions of polyvinyl alcohol, and the VL grade formulations are based on water-alcohol solutions of polyvinylbutyral and its modifications. The decontaminating components in these products are mineral acids (nitric, orthophosphoric), orga- 
nic acids (citric, acetic), complexing agents (EDTA, OEDFA, etc.), and surface-active substances (OP-7, OP-10).

The VA and VL grade compositions are manufactured by Dreko Co. Ltd. (Khimmed Co. Ltd.) and Dial Co. Ltd.; according to data from Dreko Co. Ltd., the declared storage period is one year. It should be specifically noted that the VA and VL products are the only commercially available decontamination film-forming agents recommended for use at NPPs (OST 95 10562-2001).

Decontaminating properties of the ZPS-1M, VA(501-504), and VL(501-504) compositions deserve special consideration. On the one hand, these compositions have undergone the required testing. As shown by the test results, the decontamination factor for the surfaces contaminated with beta nuclides is not less than 100 , and for those contaminated with alpha nuclides is not less than 1000. It should be however noted that the test procedures described in the respective regulatory document (GOST R 50773-95) does not suggest a test to check the removal efficiency for fixed RC. This fact has been noted more than once (Koryakovskiy et al. 2012, 2012a, 2014, 2016).

Tests of the VA and VL grade compositions performed on stainless steel samples at St. Petersburg State Institute of Technology (Technical University) showed a very low efficiency of the fixed RC removal: the decontamination factors (DF) for cobalt-60, strontium-90, and cesium-137 did not exceed 5 to 6 .

Despite the fact that the commercially available film-forming compositions are not good in removing fixed contamination, the actual potential of this technology is much greater. In particular, this has been proved by a positive experience of using polymer compositions in the Chernobyl accident response activities (Table 1).

As follows from the table, the efficiency of dry decontamination (i.e. using different removable coatings) turned out to be the highest for the overwhelming majority of contaminated materials (Stepanov et al. 2006). Besides, additional tests of the VA and VL compositions have shown that modifying them by various additives make it possible, in a number of cases, to increase DF greatly as compared with the base product.

Table 1. Decontamination efficiency for external surfaces of building materials in the city of Pripyat based on different methods (Stepanov et al. 2006).

\begin{tabular}{|c|c|c|c|}
\hline \multirow{2}{*}{$\begin{array}{c}\text { Decontamination ma- } \\
\text { terial }\end{array}$} & \multicolumn{3}{|c|}{$\begin{array}{l}\text { Decontamination factor DF } \\
\text { Decontamination method }\end{array}$} \\
\hline & foam & jet & $\begin{array}{c}\text { dry } \\
\text { (film) }\end{array}$ \\
\hline Glass & 30 & 30 & $80-100$ \\
\hline Concrete, not painted & 10 & 20 & 10 \\
\hline Concrete, painted & 15 & 18 & 20 \\
\hline Tiles, encaustic & 20 & 25 & $60-80$ \\
\hline Iron, painted & 20 & 25 & $70-90$ \\
\hline Iron, galvanized & 20 & 25 & $70-90$ \\
\hline Roofing, bitumen & 2 & 5 & $70-90$ \\
\hline Wood, painted & 15 & 25 & $60-80$ \\
\hline Asphalt & 2 & 5 & $30-40$ \\
\hline
\end{tabular}

Taken together, the above facts have provided the sufficient basis for continuing research work with the VL and VA compositions.

Metal samples, to which fixed contamination was applied, were tested. The following was studied in the process of research:

- techniques of the RC application to samples;

- RC fixation strengthening methods;

- methods to inject modifying additions;

- techniques for removing polymer compositions;

- stability of modified compositions, etc.

The best results were obtained with the VL grade compositions. Fig. 1 presents decontamination factors for steel samples (with fixed RC) decontaminated with a modified VL-501 composition. The numbers on the horisontal axis indicate the additives for which an enhanced decontaminating action was recorded.

Much better decontamination properties of compositions were also shown on carbon steel samples for the VL compositions modified with oxyethylidenediphosphonic acid and aluminum chloride. In particular, the addition of aluminum chloride made it possible to increase greatly the efficiency of decontamination for samples with a layer of corrosion products: DF increased by a factor of 28 to 35 as compared with the initial composition.

However, despite the positive results, there are certain issues remaining regarding the removable coatings. Thus, bringing modified compositions into manufacture requires additional testing to identify the effects the additives have on the storage period and other characteristics of compositions. The problem of the composition viscosity control also needs to be resolved: the batches of products used for testing had a very low viscosity: that's why they could be used only on smooth horizontal surfaces since even a minor angle (of about 2 to $3^{\circ}$ ) led to the liquid compositions flowing down by gravity.

Besides, there was a strong adhesion of the formed film observed in some cases during the application of polymer compositions to rough surfaces and to rusty metals which prevented the removal of the dried coating. As a rule, the reinforcement of films (using gauze and other materials) made it possible to solve the problem but it was found that this technique often led to a reduced DF.

Further efforts to find alternative ways for the fixed contamination removal have led to the development of a new technology protected by a Russian Federation patent (RF Patent No. 2586967).

In the proposed method, a composite material is used for the RC removal. Its base component is a sorbent saturated in advance with the decontamination solution. The sorbent is retained between two layers, a lower one, moisture-permeable, that contacts the contaminated surface and the upper one which is moisture-proof (Fig. 2).

This method developed by SPSIT(TU) combines the advantages of film decontamination and decontamination 


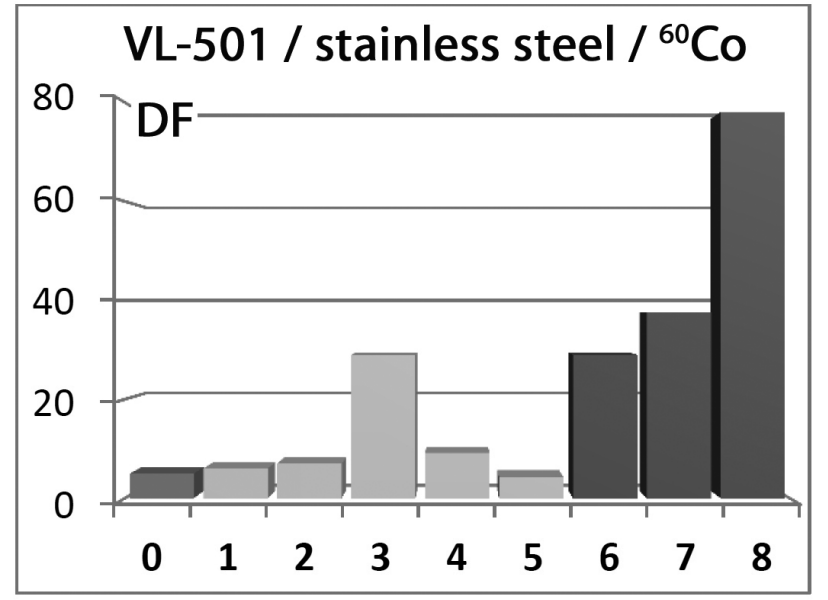

a)

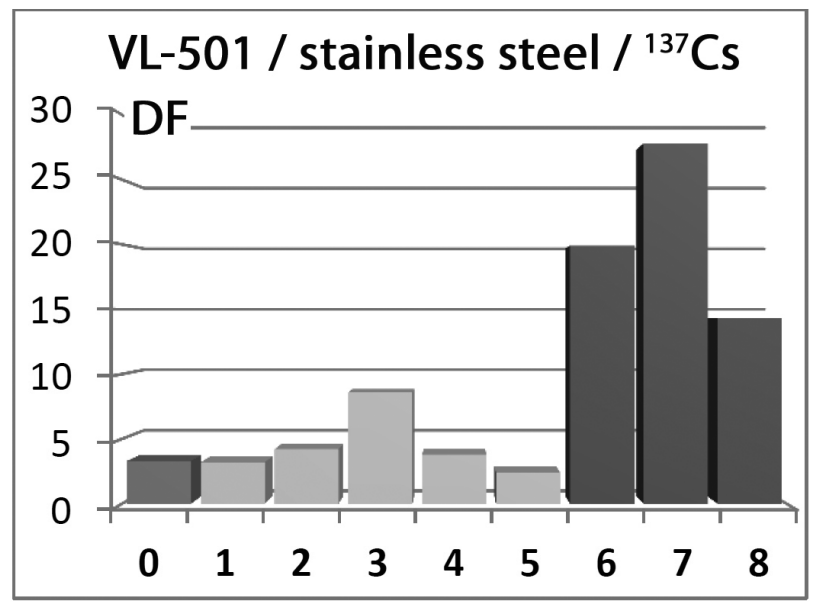

c)

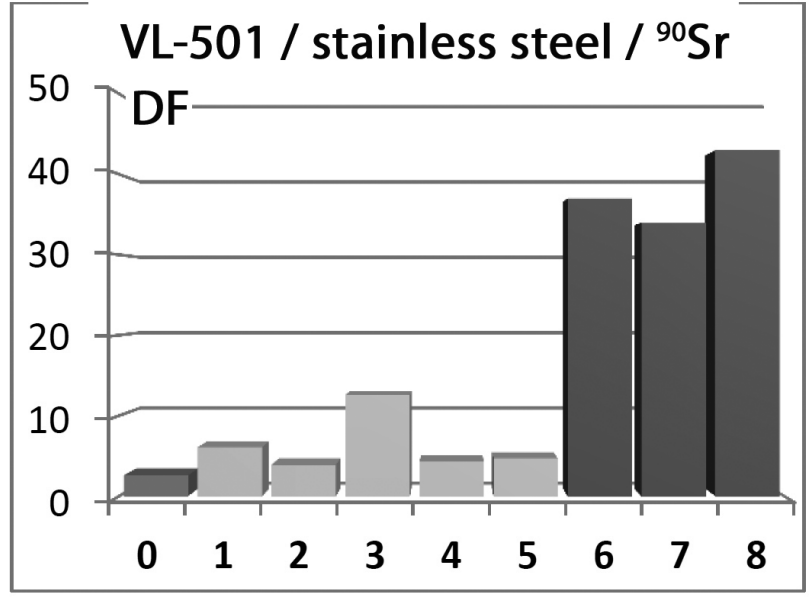

b)

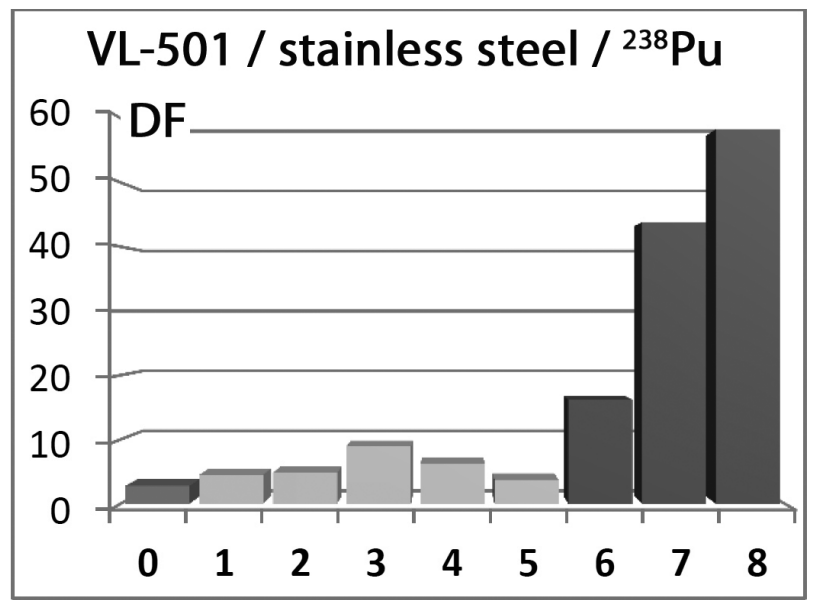

d)

Figure 1. Decontamination factors (DF) for the VL-501 stainless steel samples contaminated with radioisotopes. Modifications of the VL-501 composition: 0 - no; 1 - oxalic acid; 2 - Trilonom-B; 3 - citric acid; 4 - nitric acid; 5 - hydrochloric acid; 6 - ammonium chloride; 7 - aluminum chloride; 8 - oxyethylidenediphosphonic acid

with etching pastes (gels) with no drawbacks inherent to either of the technologies (see above).

After being saturated with the decontamination solution, the composite material is applied to the contaminated surface followed by a time delay. In the process of the solution-surface interaction, radionuclides transform to a liquid form and diffuse to the sorbent layer.

The lower layer, which is a woven or nonwoven moisture-permeable material, provides the contact of surface with the decontamination solution and the further transport of dissolved radionuclide forms into the sorbent. Thanks to the upper moisture-proof layer, the solution evaporation rate decreases radically; that's why the time of interaction between decontamination agent and contamination can be increased up to 10 to 15 days (for aqueous solutions).

Together, the lower layer and the upper layer ensure that the material is intact (so they prevent, if present, the crumbling of the sorbent and exclude the need for additional ope- rations to collect it) and make it possible to apply and remove the composite material rapidly as integral sheets or rolls.

Dehydrating supersorbents, representing polymer materials based on acrylic (methacrylic) acid and/or their derivatives and salts, as well as fluff pulp, were used as the sorbents in the experiments. But it is evident that any material capable to absorb and retain aqueous solutions with a liquid modulus of 8 to 12 and more can be used as the sorbent (the liquid modulus is understood here as ratio of the decontamination solution mass to the dry sorbent mass).

Experiments were conducted with new composite material using carbon steel, stainless steel and plastic samples.

The best decontamination results were achieved on metal surfaces.

The following acidic formulas of water solutions were used as the decontamination agents inorganic acids $(\mathrm{HCl}$, $\mathrm{HBF}_{4}, \mathrm{HNO}_{3}, \mathrm{H}_{3} \mathrm{PO}_{4}, \mathrm{H}_{2} \mathrm{SO}_{4}$ );

- organic acid (oxalic acid, citric acid, and others). 


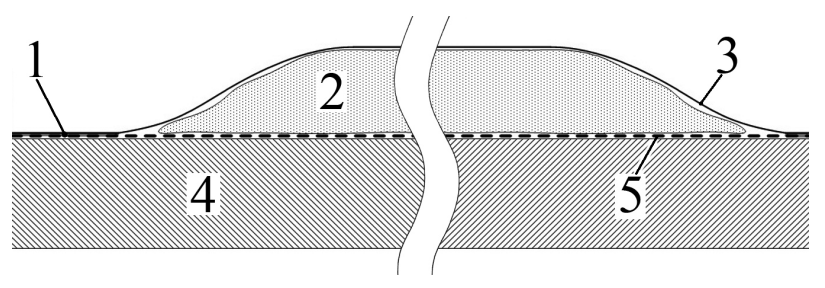

Figure 2. Structure of a three-layer composite material for decontamination: 1 - upper and lower layer connection (bonding or piercing); 2 - sorbent; 3 - upper moisture-proof layer; 4 decontaminated material; 5 - lower moisture-permeable layer.

- The concentration of the reagents changed in a broad range (from 2 to $20 \mathrm{wt}$ \%). To reinforce the action, $\mathrm{H}_{2} \mathrm{O}_{2}, \mathrm{NaF}$ and complexing agents (Trilon-B, diethylenetriaminepentaacetic acid, oxyethylidenediphosphonic acid, sodium hexamethaphosphate) were added to some of the formulas.

Acidic solutions were chosen for the metal decontamination because of fact that the fixed RC of metals normally has a bond with the oxide film on their surface, and the depth of the penetration, if any, rarely exceeds 10 to $20 \mu \mathrm{m}$. Accordingly, the prolonged action of an acidic formulation leads to the dissolution of corrosion products and the upper metal layer; and radionuclides go to a liquid phase and then diffuse into the sorbent layer.

It can be suggested that the driving force for the diffusion in this case is the gradient of concentration; the final stage in the interaction is the uniform distribution of radionuclides across the sorbent volume. Regarding this, the decontamination process can be considered completed when the radionuclide transport from the surface into the sorbent layer stops.

Concentration of decontaminating reagents, the thickness, density and chemical stability of the corrosion product layer on the metal surface, and the sorbent layer thickness are some of the factors that affect the process. In the mentioned experiments, the equilibrium time was normally 4 to 7 days.

By the time the equilibrium is achieved, radionuclides concentrate largely in the sorbent layer; a small share of the activity is retained in the thin film of the decontaminating agent that remains on the surface. The decontamination factor right after the composite material removal is about several tens: DF depends on the decontaminating agent mass in the sorbent and on the quantity of the liquid phase remaining on the surface (this quantity depends on the degree of the adhesion of liquid to the surface).

The decontaminating agent residues are recommended to be removed using a suitable absorbent material (a viscous fabric was used in the experiments); this auxiliary operation is performed rather quickly. When a floor is decontaminated, the residual liquid can be collected in a traditional way (using professional mops) and drained through the leakage collection system.

The removal of the decontamination solution residues makes it possible to improve considerably the metal decontamination efficiency and to increase DF to the values of 150 to 500. Such high figures are achieved due to the fact that the $\mathrm{RC}$ remaining on the surface after the sorbent removal are in a movable and unfixed form and can be therefore easily removed. Accordingly, if the initial values of the beta emitting nuclide surface activity are 60 to $200 \mathrm{~Bq} / \mathrm{m}^{2}$, the metal can be cleaned by the proposed method to the level of contamination that permits the unrestricted use of the metal (OSPORB-99/2010).

Therefore, the composite material saturated with a decontamination solution has a dual function: it collects most of the activity that is penetrated by a diffusion mechanism into the sorbent layer to be removed, and renders radionuclides movable which facilitates considerably the removal of residual $\mathrm{RC}$.

It is possible to increase DF greatly with no additional wiping if sorbents capable to retain radionuclides in an ion form (through a chemical sorption mechanism) are added into the "main" sorbent. It should be however taken into account that practically all known sorbents are incapable to retain radionuclides in strongly acidic environments which required to provide the best kinetics of the oxide film dissolutions. Therefore, an increase of the efficiency by chemical binding of isotopes in the sorbent layer seems to be unlikely for the acidic envoronments.

After the decontamination is over, it is recommended that the surface should be wiped using, e.g., a 2 to $5 \%$ caustic soda solution to prevent the contaminated metal corrosion.

Besides metals, polymer samples were also used to test the proposed composite material. A floor coating of a chloride-polyvinyl plastic compound of grade 57-40 was taken as a polymer broadly used at radioactively hazardous facilities. The following solutions were tried to decontaminate it by technique described above:

- aqueous inorganic acids (with or with no addition of auxiliary reagents);

- aqueous organic acids (with or with no addition of auxiliary reagents);

- based on alkali reagents with addition of complex formers.

The best results were obtained for the solution with the following composition:

$$
5 \% \mathrm{HNO}_{3}+0.2 \% \mathrm{H}_{2} \mathrm{C}_{2} \mathrm{O}_{4}+0.2 \% \mathrm{NaF}
$$

This formulation was used to decontaminate a laboratory room with a floor coating made of plastic compound contaminated by alpha emitting nuclides (predominantly plutonium-239).

The efficiency of decontamination turned out to be lower than for non-painted metals with fixed contamination. This was an expected result since plutonium, as known, tends to penetrate into the depth of polymers, while the aqueous formulation interacts just with surface of plastic substance. The decontamination factor changed in broad range, from a few up to tens, with the maximum DF va- 
lues of about 60-70. On the whole, however, the decontamination activities made it possible to improve radiological situation in the room considerably, and most of the area had the RC levels reduced to permissible values.

To be successfully implemented, a new decontamination technology evidently requires the flowchart describing how to handle the secondary waste generated. As to the method discussed above, radwaste represents sheets (rolls) of the composite material (containing the radioactive decontamination solution) that have been removed from the surface.

It would be reasonable that the decontamination solution to be pressed (squeezed) out of the sorbent at first stage; it is proposed that then "drained" sorbent and liquid radioactive waste (LRW) should be treated separately. It should be taken into account that the liquid phase cannot be squeezed out fully, and the "drained" sorbent will contain residues of the decontamination solution. This requires that methods used to treat both the sorbent and LRW to be harmonized with the initial composition of the decontamination solution.

The LRW formed by pressing can be combined with other liquid waste, specifically with solutions drained from baths for submerged decontamination. Pressed out sorbents can be processed using either low- or high-temperature technologies.

The following "cold" technologies can be identified:

- drying with subsequent containerization;

- grouting of preliminarily neutralized sorbents.

Low-temperature methods offer an advantage: they require rather simple equipment; specifically, the following "cold" processing flowchart with the final grouting can be proposed (Fig. 3).

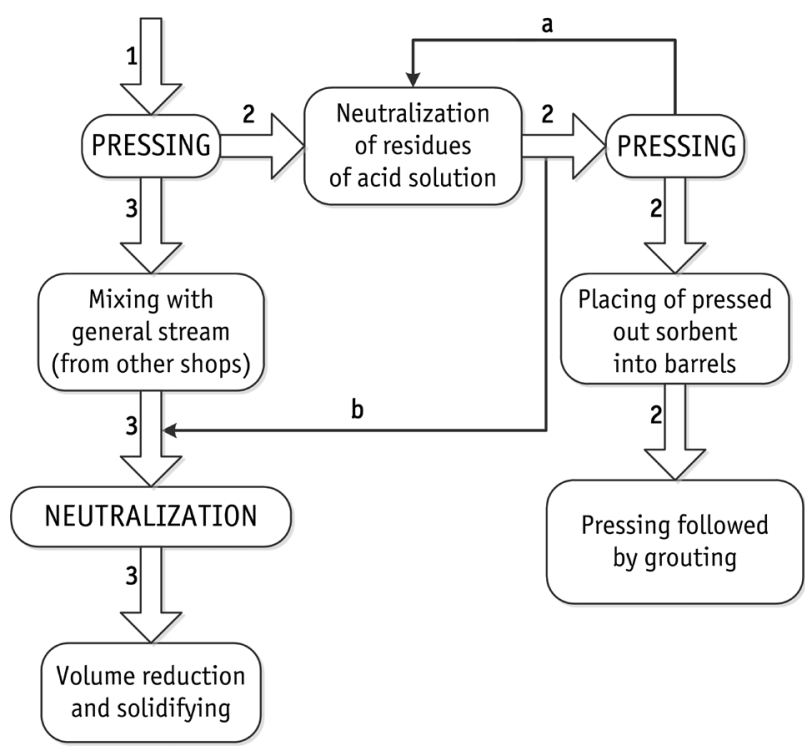

Figure 3. Option of the flowchart to process the composite material removed from the decontaminated surface: 1 - spent sorbent saturated with radioactive solution; 2 - pressed out (squeezed) sorbent; 3 - acidic decontamination solution; a - return of neutralizing solution for reuse; $b$ - drain of neutralizing solution
1. Spent sorbent is fed for pressing out (squeezing).

2. After being squeezed, the sorbents containing residues of the acid decontamination solution are submerged into the neutralizing solution and are then pressed again.

3. After the repeated squeezing, the sorbents are placed in drums, pre-compressed and grouted. The prior pre-compression (compaction) makes it possible to prevent the swelling of sorbent (as a result of interaction with water contained in the grout).

4. The spent acidic decontamination solution drained after the initial sorbent pressing is combined with other LRW arising in other decommissioning activities.

5. The combined stream is neutralized and processed using the technology which suits the LRW chemical composition (e.g., evaporation for the volume reduction) and then is fed grouting.

An alternative to "cold" technologies is "hot" processing technologies (pyrolysis, incineration). They make it possible to reduce considerably the RW amounts but, on the other hand, they represent much more expensive conditioning processes. Besides, high-temperature treatment requires measures to be taken to handle hot off-gases containing volatile radionuclides and (quite likely) toxic compounds.

Along with high DF values, one of the key advantages offered by the proposed decontamination method is that the duration of the decontamination activities (performed by operator) is drastically reduced: the sorbent can be laid as part of the composite material and removed with a total of 6.0 to 7.5 seconds per $\mathrm{m}^{2}$ spent (for the option when the material is rolled out and rolled back). Therefore, using the considered technique makes it possible to reduce the time for the decontamination media to be applied and removed by a factor of 11 to 16 (as compared with the known decontamination technique by pastes or gels).

Besides, unlike polymer coatings that sometimes are hard to remove (Stepanov et al. 2006), the proposed technology ensures that the sorbent is removed from the surface rapidly and as single piece in almost all situations (to the exception of cases when the lower surface-contacting layer dries). Such cases were, however, observed only with a very long hold time of interaction (about 10 days or more).

\section{Conclusions}

1. An analysis of the methods used for in-situ chemical decontamination has shown that some of the considered methods demonstrate, not infrequently, a very low efficiency in removal of fixed RC. When more efficient techniques are used, decontamination activities normally require a longer time which entails enhancement of personnel exposure doses.

2. Modifying commercially available film-forming compositions of the VA and VL grades makes it possible in some cases to increase DF greatly (by a factor of 5 to 35), as compared to the initial com- 
positions. Further studies are however required to determine the influence the additives have to the storage life and other performance characteristics of compositions.

3. Using a new composite cover material for decontamination based on sorbents saturated with a de- contamination solution makes it possible to achieve rather high decontamination factors for metals (from 150 to 500). This method can be also used for polymer and other nonmetal materials.

\section{References}

- Ampelogova NI, Simanovskiy YuM, Trapeznikov AA (1982) Decontamination in Nuclear Power. Energoizdat Publ., Moscow, 256 pp. [in Russian]

- GOST R 50773-95 (1995) Protective Polymer Coatings for Improving the Radiation Situation. Methods of Determining the Decontamination Factor and the Protective Action Time. Gosstandart Rossii Publ., Moscow, 20 pp. [in Russian]

- Koryakovskiy YuS, Akatov AA, Doilnitsyn VA (2014) Decontamination by film-forming compositions: advantages and limitations, applicability during decommissioning. Proc. of the $9^{\text {th }}$ Int. Nucl. Forum "Safety of Nuclear Technologies: Emergency Preparedness and Response”. NOU DPO “TsIPK Rosatoma” Publ., St. Petersburg, 232-235. [in Russian]

- Koryakovskiy YuS, Akatov AA, Maksimova UM, Doilnitsyn VA, Matveev SA (2012) Development of new removable polymer coatings for decontamination. Proc. of the Sci. and Pract. Conf. dedicated to the $184^{\text {th }}$ anniversary of the establishment of Saint-Petersburg State Institute of Technology. SPbGTI(TU) Publ., St. Petersburg, 189. [in Russian]

- Koryakovskiy YuS, Akatov AA, Matveev SA (2012a) Modification of Removable Polymer Coatings Used for Decontamination. Proc. of the $2^{\text {nd }}$ Sci.-Tech. Conf. of Young Scientists "Nedelya nauki-2012". SPbGTI(TU) Publ., St. Petersburg, 141. [in Russian]

- Koryakovskiy YuS, Doilnitsyn VA, Akatov AA (2010) Decontamination: Ensuring Radiation Safety at Nuclear Industry Enterprises. Training Guide. SPbGTI(TU) Publ., St. Petersburg, 151 pp. [in Russian]

- Koryakovskiy YuS, Doilnitsyn VA, Akatov AA (2016) Use of improved film-forming polymer compositions for enhancing the efficiency of metal decontamination. Proc. of the Sci. and Pract. Conf. "Topical Issues of Nuclear Chemical Technologies and Environmental Safety”. SevGU Publ., Sevastopol, 142-145. [in Russian]

- Koryakovskiy YuS, Doilnitsyn VA, Akatov AA, Matveev SA (2014a) Removable polymer coatings for decontamination: achievements and inventions, application, availability of technologies (review). Izvestiya Sankt-Peterburgskogo gosudarstvennogo tekhnologicheskogo instituta (tekhnicheskogo universiteta) 25(51): 68-79. https://doi. org/10.15217/issn1998984-9.2014.25.68

- Kritskiy VG (2010) Activities Aimed to Reduce the Dose Rate in the NPP Primary Circuit Rooms. Preprint. GI VNIPIET Publ., St. Petersburg, 187 pp. [in Russian]

- OSPORB-99/2010 (2014) Basic Health Regulations for Ensuring Radiation Safety: Sanitary Standards and Rules, SP 2.6.1.2612-10. UralYurIzdat Publ., Yekaterinburg, 108 pp. [in Russian]

- OST 95 10562-2001 (2002) Protective Polymer Coatings for Nuclear Power Plants. Part 1. Removable Protective Polymer Coatings. General Requirements, Application and Selection. VNIIPO Publ., Moscow, 32 pp. [in Russian]

- Pat. of WIPO WO2013092633, Int. cl. A61L2/232, A61L2/18 (2013) Decontamination Gel and Method for Decontaminating Surfaces by Wetting Using Said Gel, 70 pp.

- RF Patent, No. 2017244, Int. cl. G21F9/36 (1994) Method to decontaminate stainless steel surfaces, $7 \mathrm{pp}$. [in Russian]

- RF Patent, No. 2259422, Int. cl. G23/F1/28 (2005) Urea-containing agent for etching and the way of its production, $15 \mathrm{pp}$. [in Russian]

- RF Patent, No. 2586967, Int. cl. G23F1/28 (2016) Method to decontaminate contaminated metal and non-metal surfaces, $18 \mathrm{pp}$. [in Russian]

- Stepanov IK, Konstantinov YeA, Simanovskiy YuM (2006) Normalization of the Radiation Situation in the Process of the Post-Accident Recovery at the Chernobyl NPP Site. Book 4. Sosnovy Bor. Leningrad NPP Publ., 208 pp. [in Russian]

- TU 2316-219-56271024-2007 (2007) ZPS-1M3 protective polymer composition. Pigment Publ, St. Petersburg. [in Russian] http://www. pigment.ru/catalog/item/81 [accessed Feb 10, 2018]

- Vasilenko VA, Yefimov AA, Stepanov IK (2010) Technologies to Ensure Radiation Safety at NPP Sites. NIC Morintekh Publ., St. Petersburg, 576 pp. [in Russian] 